\title{
Effects of Caffeine are more Marked on Daytime Recovery Sleep than on Nocturnal Sleep
}

\author{
Julie Carrier*,1,2, Marta Fernandez-Bolanos',2, Rébecca Robillard ${ }^{1,2}$, Marie Dumont', Jean Paquet', \\ Brahim Selmaoui' and Daniel Filipini' \\ 'Centre d'étude du sommeil et des rythmes biologiques, Hôpital du Sacré-Cœur de Montréal, Montreal, QC, Canada; ${ }^{2}$ Centre de recherche \\ en neuropsychologie et en cognition, Département de psychologie, Université de Montréal, Montreal, QC, Canada
}

\begin{abstract}
Caffeine is often used to counteract sleepiness generated by sleep deprivation, jet lag, and shift-work, and is consumed at different times of day. Caffeine also has effects on sleep. However, little is known about the interaction between sleep deprivation, circadian timing, and caffeine consumption on sleep. In this study, we compared the effects of caffeine on nocturnal sleep initiated at habitual circadian time and on daytime recovery sleep. Thirty-four moderate caffeine consumers participated in both caffeine (200 mg) and placebo (lactose) conditions in a double-blind crossover design. Seventeen subjects followed their habitual sleep-wake cycle and slept in the laboratory during the night (Night), while 17 subjects were sleep deprived for one night and recovery sleep started in the morning (DayRec). All subjects received a capsule of $100 \mathrm{mg}$ of caffeine (or placebo) $3 \mathrm{~h}$ before bedtime, and the remaining dose I $\mathrm{h}$ before bedtime. Compared to placebo, caffeine lengthened sleep latency, increased stage I, and reduced stage 2 and slow-wave sleep (SWS) in both groups. However, caffeine reduced sleep efficiency more strongly in the DayRec group, and decreased sleep duration and REM sleep only in that group. The stronger effects of caffeine on daytime recovery sleep compared to nocturnal sleep are probably the consequence of the combined influence of increasing circadian wake propensity drive and the dissipation of homeostatic sleep pressure. We propose that the reduction of SWS by caffeine during daytime sleep increases the impact of the circadian wake signal on sleep. These results have implications for individuals using caffeine during night time.
\end{abstract}

Neuropsychopharmacology (2007) 32, 964-972. doi: I0.1038/sj.npp. I 30I 198; published online 23 August 2006

Keywords: caffeine; sleep; circadian rhythms; adenosine; sleep deprivation

\section{INTRODUCTION}

Caffeine is considered to be the most widely used psychoactive drug today. Consumption from all sources can be estimated to be approximately $210-238 \mathrm{mg} / \mathrm{day} /$ person in Canada and the US (Barone and Roberts, 1996). Studies have shown that the ingestion of $100-400 \mathrm{mg}$ of caffeine in the evening increases sleep latency and sleep motility, decreases sleep consolidation, and reduces the amount of slow-wave sleep (SWS) (Bonnet and Arand, 1996; Brezinova, 1974; Karacan et al, 1976; Kelly et al, 1997; Landolt et al, 1995). Studies show that the most probable biochemical mechanism of the effects of caffeine on sleep is the blockade of $\mathrm{A} 1$ and $\mathrm{A} 2 \mathrm{a}$ adenosine receptors (Fredholm et al, 1999).

*Correspondence: Dr J Carrier, Centre d'étude du sommeil et des rythmes biologiques, Hôpital du Sacré-Cœur de Montréal, 5400 boul. Gouin Ouest, Montréal, QC, Canada H4J IC5, Tel: + I 5I4338 2222 ext. 3124, Fax: + | 514338 2531, E-mail: julie.carrier. |@umontreal.ca Received 9 May 2006; revised 10 July 2006; accepted 19 July 2006 Online publication: 21 July 2006 at http://www.acnp.org/citations/ Npp072 106060308/default.pdf
Moderate doses of caffeine reduce reaction time on performance tasks, improve subjective alertness, and diminish self-reported fatigue and sleepiness (Landolt et al, 2004; Reyner and Horne, 2000; Van Dongen et al, 2001; Walsh et al, 1990; Warburton, 1995; Wyatt et al, 2004). Consequently, caffeine is consumed at different times of the day and night to help counteract the effects of sleep deprivation and circadian phase misalignment (jet lag, night work) on vigilance. However, little is known about the interaction between sleep deprivation, circadian timing, and caffeine consumption on sleep. Two animal studies showed that caffeine reduces sleep depth and sleep continuity of recovery sleep, following sleep deprivation (Schwierin et al, 1996; Wurts and Edgar, 2000). In humans, one study has shown minimal effects of caffeine on nocturnal recovery sleep after $64 \mathrm{~h}$ of sleep deprivation (Beaumont et al, 2005). Using quantitative sleep electroencephalogram (EEG), a recent study by Landolt et al (2004) has shown that the administration of two doses of caffeine during a 40 -h sleep deprivation significantly reduces EEG power in the $0.75-$ $2.0 \mathrm{~Hz}$ band and enhances power in the $11.25-20.0 \mathrm{~Hz}$ range relative to placebo during recovery night. Only two human studies have evaluated the effects of caffeine on daytime 
recovery sleep following one night of sleep deprivation (LaJambe et al, 2005; Muehlbach and Walsh, 1995). In one study, no effect of caffeine on daytime recovery sleep was found (Muehlbach and Walsh, 1995), while in the other study caffeine reduced SWS and sleep duration, and increased wakefulness during recovery sleep (LaJambe et al, 2005). These conflicting results may be explained by different caffeine levels in the body at bedtime, since in the first study caffeine was administered more than $8 \mathrm{~h}$ before daytime recovery sleep, while in the second study the last dose of caffeine was taken $2 \mathrm{~h}$ before sleep.

According to contemporary models of sleep-wake cycle regulation, the interaction of homeostatic and circadian processes regulates the sleep-wake cycle (Achermann et al, 1993; Borbély and Achermann, 2000; Broughton, 1998; Daan et al, 1984). The homeostatic process represents the accumulation of sleep pressure with increasing time awake and its dissipation during a sleep episode. The circadian process represents the rhythmic variation of sleep and wake propensity over $24 \mathrm{~h}$. Studies have shown important differences in sleep variables between habitual night time sleep and daytime sleep initiated after a sleep deprivation (Akerstedt, 1984; Gaudreau et al, 2001; Weitzman and Kripke, 1981). The interaction between the homeostatic and the circadian processes underlies these differences. Hence, elevated homeostatic sleep pressure induced by sleep deprivation accounts for a higher amount of SWS in daytime recovery sleep compared to night time sleep (Achermann et al, 1993). Despite the deprivation of sleep, daytime recovery sleep is more fragmented and shorter than night time sleep because it is initiated at a point in the circadian rhythm when the biological clock is providing an increasing wake signal (Dijk and Czeisler, 1994). The higher amount of wakefulness during daytime recovery sleep compared to night time sleep is more prominent at the end of the sleep episode, at the expense of stage 2 and REM sleep (Gaudreau et al, 2001).

In a previous report, we suggested that the amount of SWS or homeostatic pressure is critical for the ability to maintain sleep when the biological clock is promoting wakefulness, such as during daytime (Gaudreau et al, 2001). According to this hypothesis, the amount of SWS helps sleep to override the increasing circadian wake signal that is found during daytime. Consequently, the effects of caffeine on sleep consolidation during daytime recovery sleep should be stronger compared to nocturnal sleep, because the reduction of SWS reduces the ability to maintain sleep during daytime. The aim of the present study was to compare the effects of caffeine on nocturnal sleep initiated at habitual circadian time as well as on daytime recovery sleep. Since caffeine decreases SWS, we predicted that the effects of caffeine on the sleep consolidation parameters would be stronger when administrated before daytime recovery sleep than before habitual nocturnal sleep.

\section{EXPERIMENTAL PROCEDURE}

\section{Subjects}

Thirty-four moderate caffeine consumers (equivalent to one to three cups of coffee per day) participated in these studies. All subjects were in good health according to their medical history. They were all non-smokers, and were not consuming any drug or medication that affect the sleep-wake cycle. Blood sample analysis (complete blood count, serum chemistry including hepatic and renal functions, levels of prolactine, levels of testosterone in men, and levels of estrogen, FSH and $\mathrm{LH}$ in women) and urinalysis results were verified by a physician for any significant abnormalities. All subjects were negative on a THC screening test. Other exclusion criteria were the presence or history of a psychiatric or neurological illness, a body mass index (BMI) greater than 28.5, and transmeridian travelling or night work in the three months before the study.

None of the subjects reported any sleep problems. Each participant underwent a polysomnographic (PSG) screening night in the laboratory, during which EEG, electromyogram (EMG), and electrooculogram (EOG) were recorded. A nasal/oral thermistor and EMG leg electrodes were also used to screen for breathing and sleep movement disorders. The presence of sleep disturbances such as sleep apneas and hypopneas (index per hour $>10$ ), periodic leg movements (index per hour $>10$ ), prolonged sleep latency ( $>30 \mathrm{~min}$ ), or low sleep efficiency $(<85 \%)$ resulted in the exclusion of the participant. Subjects were instructed to abstain from alcohol and medication during the laboratory component of the experiment.

While the study included both pre- and postmenopausal women, perimenopausal women as well as women using hormonal contraceptives or receiving hormonal replacement therapy were excluded. Premenopausal women reported having regular menstrual cycles (25-32 days) during the year preceding the study, had no vasomotor complaints (ie hot flashes, night sweats) and showed low FSH levels $(<20 \mathrm{iU} / \mathrm{L})$. These women started the laboratory sessions during the follicular phase of their menstrual cycle. All postmenopausal women reported an absence of menses during the past year, and their FSH levels were above $20 \mathrm{iU} / \mathrm{L}$. This research project was approved by the hospital's ethics committee. All subjects signed a consent form providing detailed information about the nature, purpose, and potential risks of the study, and received financial compensation for their participation.

\section{Research Protocol}

Subjects were divided into two groups (matched for age and gender): (1) Night group ( $n=17 ; 10$ women, 7 men; mean age $=37.2$ years, $\mathrm{SEM}=3.5)$ and (2) DayRec group $(n=17$; 10 women, 7 men, mean age $=39.9$ years, $\mathrm{SEM}=3.8)$. BMI did not differ between the two groups (Night group: mean $=23.02, \quad S E M=0.7 ;$ DayRec group: mean $=23.6$, $\mathrm{SEM}=0.7$; NS). Figure 1 illustrates the research protocol for each group. Procedures were similar for the two groups. Exceptions were the time of day of caffeine/placebo administration, and the timing of the sleep episode. Subjects in the Night group followed their habitual sleep-wake cycle and slept in the laboratory during the night, while subjects in the DayRec group were sleep deprived for one night $(25 \mathrm{~h}$ of wakefulness) and recovery sleep started in the morning, $1 \mathrm{~h}$ after their habitual wake time. All subjects participated in both the caffeine $(200 \mathrm{mg}$ ) and placebo (lactose in identical capsules) conditions, with a double-blind crossover design. This dose of caffeine is considered to be 


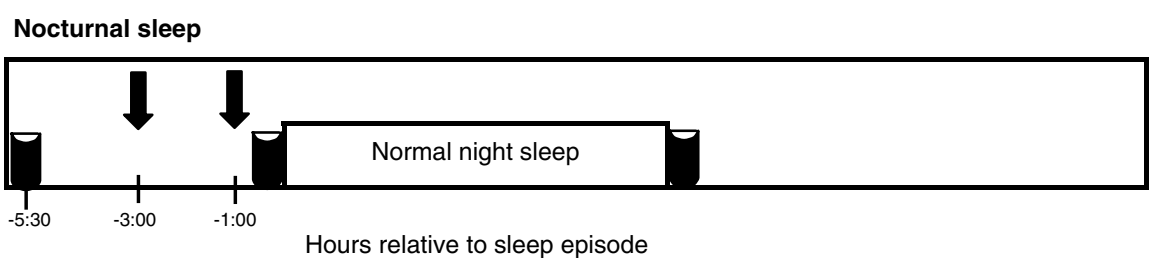

Daytime recovery sleep
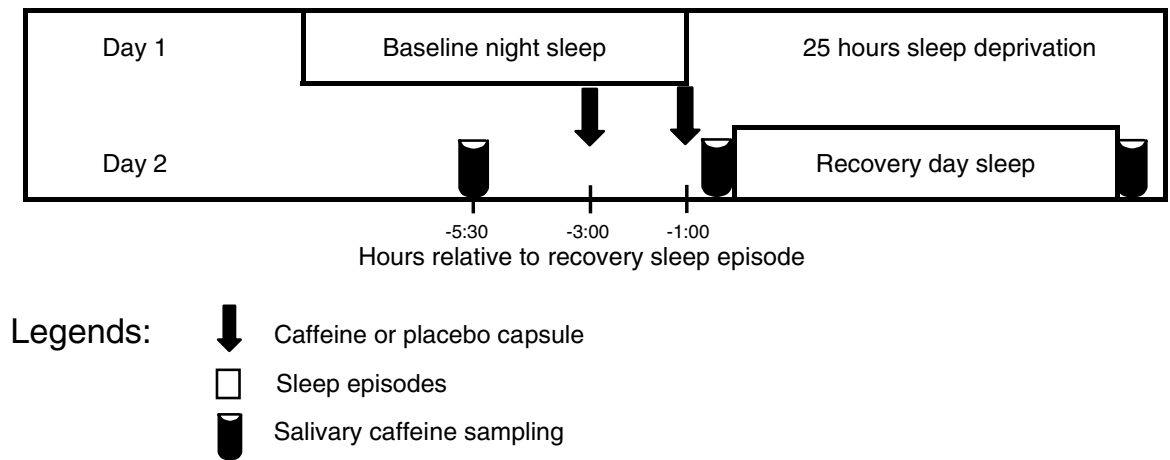

Figure I Schematic representation of the research protocol for the Night group and the DayRec group.

moderate, and is known to produce significant effects on sleep (Landolt et al, 1995). Subjects received one capsule containing either caffeine $(100 \mathrm{mg})$ or placebo $3 \mathrm{~h}$ prior to their bedtime, and the remaining dose of caffeine $(100 \mathrm{mg})$ or placebo was administered $1 \mathrm{~h}$ before bedtime.

One week prior to each laboratory condition, subjects were instructed to keep a regular sleep-wake pattern within $30 \mathrm{~min}$ of their habitual sleep-wake schedule, and to maintain their habitual caffeine intake. Subjects kept the same sleep-wake schedule for each condition. During this time, they were asked to complete the French version of the 'Pittsburgh Sleep Diary' (Monk et al, 1994), and to report the amount as well as the different caffeinated products they had consumed (ie coffee, tea, chocolate, etc.). The mean number of milligrams of caffeine consumed per day was approximated for each subject according to the following criteria: $250 \mathrm{ml}$ of coffee $=100 \mathrm{mg}$ caffeine; $250 \mathrm{ml}$ of tea $=50 \mathrm{mg}$ of caffeine; $250 \mathrm{ml}$ of cola $=35 \mathrm{mg}$ of caffeine; $10 \mathrm{~g}$ of chocolate $=5 \mathrm{mg}$ of caffeine. Subjects also reported alcohol intakes on the sleep diaries. Mean daily alcohol intake for the week prior to each laboratory session was low and did not differ between the groups (Night group: mean $=0.6$ drinks, $\mathrm{SEM}=0.1$; DayRec group: mean $=0.7$, $\mathrm{SEM}=0.2 ; \mathrm{NS}$ ). On experimental days, subjects were required to abstain from alcohol, medication, and naps, and were permitted to maintain their habitual caffeine consumption in the morning, in order to prevent potential effects of caffeine withdrawal. Starting at noon, subjects stopped consuming caffeinated beverages and foods.

Subjects in the Night group came to the chronobiology laboratory for two sessions. Each session (caffeine, placebo) was separated by 6-9 days. Subjects arrived in the laboratory at the end of the afternoon. Bed and wake times in the laboratory were based on the subject's habitual sleepwake cycle, averaged from their sleep diary. Subjects in the DayRec group came to the laboratory for two sessions, which were separated by a month to make sure that the effects of sleep deprivation were dissipated and to study women in a similar menstrual phase in both conditions. For each session (caffeine, placebo), subjects came to the laboratory for one baseline night. Following their departure from the laboratory in the morning, subjects performed their regular activities until the end of afternoon, at which point they came back to the chronobiology laboratory. Subjects then remained awake in bed until the next morning. A research assistant was present at all times to make sure subjects were not falling asleep. A morning recuperative sleep episode was initiated $1 \mathrm{~h}$ after their habitual wake time (following $25 \mathrm{~h}$ of wakefulness). Subjects were asked to stay in bed for their habitual sleep duration.

In both groups, subjects stayed awake in bed in a semirecumbent position in dim light $(<15$ lux $)$ from the time they arrived at the laboratory until bedtime. They were only permitted to get out from bed to go to a washroom, which was situated less than 10 feet from the bed.

\section{Measures}

Salivary caffeine concentration. Saliva was collected using the Salivette devices (Sarstedt, Inc.), and was then centrifuged and frozen immediately. Each subject provided a saliva sample $2.5 \mathrm{~h}$ before the first caffeine/placebo capsule, $5 \mathrm{~min}$ before bedtime, and $5 \mathrm{~min}$ after wake time. A rapid high-performance liquid chromatographic (HPLC) method was used for the analysis of caffeine in saliva (Alkaysi et al, 1988). The HPLC system consisted of a Spectra SYSTEM pump and a spectra SYSTEM UV detector (Thermoseparation Products Inc.). Ultrasphere $(5 \mu \mathrm{m})$ column $(250 \times 4.6 \mathrm{~mm}$, Beckman $)$ was used for the separation. The mobile phase was made of $0.05 \mathrm{M}$ ammonium acetate buffer: acetonitrile methanol $(82: 15: 3, \mathrm{v} / \mathrm{v})$. The flow rate was set to $1 \mathrm{ml} / \mathrm{min}$ and the injection volume was set at $50 \mu$ l. The detection wave length was $254 \mathrm{~nm}$. The concentrations of caffeine solution used for the standard curves were $0.5,0.25,0.125,0.1$, and $0.05 \mu \mathrm{g} / \mathrm{ml}$. Standard curves were constructed by plotting concentration $v s$ area 
under the curve. The retention time of caffeine was $5 \mathrm{~min}$. The limit detection was $0.024 \mu \mathrm{g} / \mathrm{ml}$. The intra- and interassay coefficients of variation were below $7 \%$.

PSG sleep recordings. EEG electrodes were placed according to the international $10-20$ system, using a referential montage with linked ears, chin EMG, and left and right EOG. A Grass Model 15 Neurodata system with amplifiers 15A54 (gain 10000 , bandpass $0.3-100 \mathrm{~Hz},-6 \mathrm{~dB}$ ) was used, and signals were digitized at a sampling rate of $256 \mathrm{~Hz}$ using a commercial software (Harmonie 5.1, Stellate Systems, Montreal, Canada). Sleep stages were visually scored on a computer screen (LUNA, Stellate Systems, Montreal, Canada) according to standard criteria (Rechtschaffen and Kales, 1968) that were adapted to the scoring of 20-s epochs. Sleep stage variables and the number of minutes of wakefulness were computed from sleep onset to the last epoch of sleep. Sleep efficiency was defined as (the number of minutes spent asleep/total number of minutes from sleep latency to lights on) $\times 100$. Number of minutes of wakefulness, REM sleep, and SWS sleep per third of the bedtime period (from sleep onset to lights on) were calculated.

\section{Statistical Analyses}

To evaluate group differences in salivary caffeine concentration, a three-way ANOVA with one independent factor (Group: Night and DayRec) and two repeated measures (Condition: placebo and caffeine; and Time: baseline, bedtime and wake time) were performed. Since the morning measure was missing for one subject in each group, this analysis was performed with the 32 remaining subjects.

Two-way ANOVAs with one independent factor (Group: Night and DayRec) and one repeated measure (Condition: placebo and caffeine) were performed to evaluate differences on habitual caffeine consumption (log transformed) and PSG sleep variables. To evaluate differences in sleep per third of the bedtime period, three-way ANOVAs with one independent factor (Group: Night and DayRec) and two repeated measures (Condition: placebo and caffeine; and Part of the bedtime period: $1 / 3,2 / 3,3 / 3$ ) were performed. Due to an abnormal distribution total wake time, some sleep variables were log transformed (sleep latency, REM latency, $\%$ and min stage 1 sleep, $\%$ and min SWS, $\%$ stage 2 sleep). Minutes of wakefulness, REM sleep and SWS per third of the bedtime period were squared-root transformed. Simple effects analyses were performed when significant interactions were found. $p$-values for repeated measures with more than two levels were adjusted for sphericity with Huynh-Feldt correction.

\section{RESULTS}

\section{Habitual Sleep-Wake Cycle and Caffeine Consumption}

Table 1 presents mean habitual wake time, bedtime, and approximated daily caffeine consumption in the Night and DayRec groups, for the week prior to the placebo and caffeine conditions. Means of wake times and bedtimes in the laboratory are also presented for each group and each condition. There were no significant effects for group or condition or interactions for these variables.

\section{Salivary Caffeine}

Figure 2 illustrates salivary caffeine concentrations at baseline, bedtime, and wake time in the Night and DayRec groups, for the placebo and caffeine conditions. A significant interaction between group, condition, and moment was found $(\mathrm{F}(2,60)=7.5 ; p=0.001 ; \varepsilon=1)$. At baseline, there was a significant group effect $(\mathrm{F}(1,30)=41.1 ; p<0.001)$. The Night group showed higher caffeine concentration than the

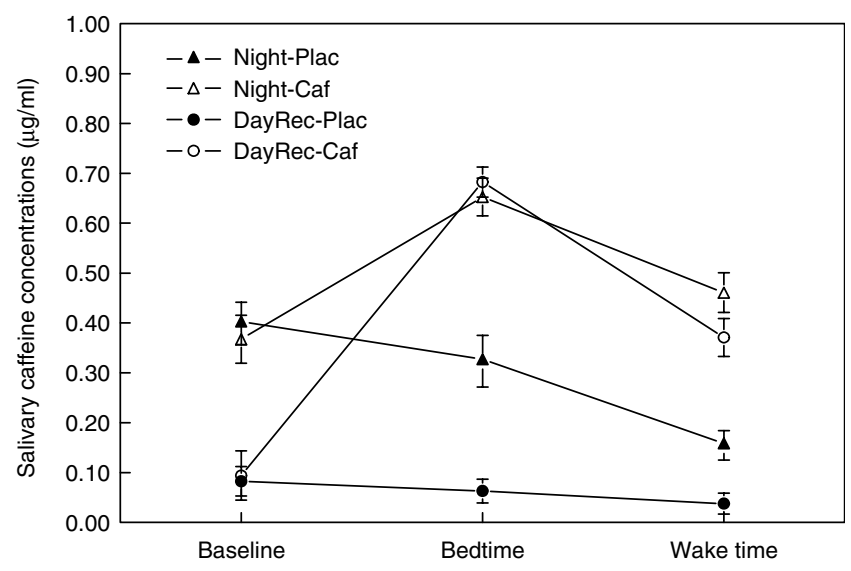

Figure 2 Caffeine concentration in saliva (mean and SEM) before capsules administration (baseline), 5 min before bedtime (bedtime), and 5 min after wake time (wake time) in the placebo and the caffeine conditions, for the Night group and the DayRec group.

Table I Diary Variables for the Week prior to Laboratory Sessions and Laboratory Wake Schedule for the Night and the DayRec Groups in the Placebo and Caffeine Conditions (Mean and SEM)

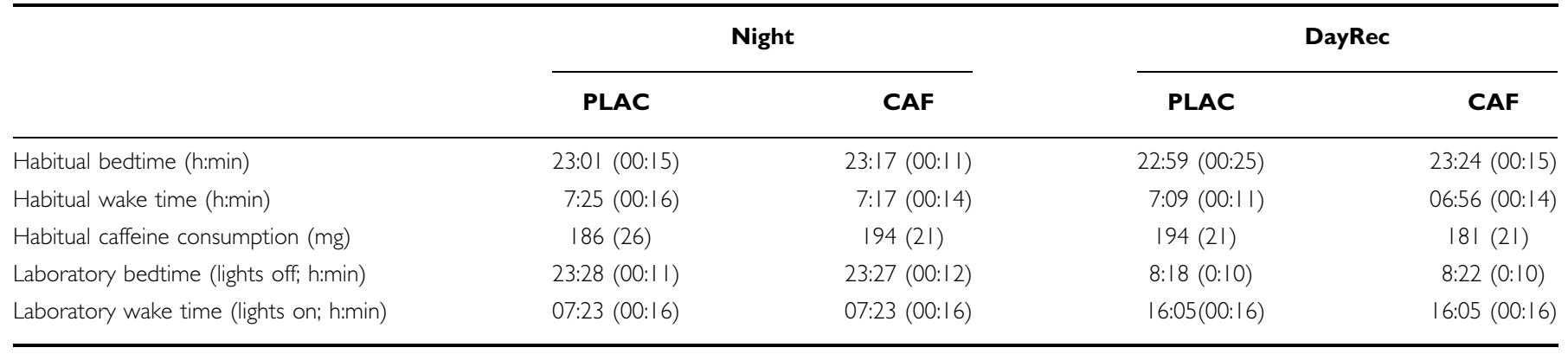


DayRec group. At bedtime, there was a significant interaction between group and condition $(\mathrm{F}(1,30)=25.4$; $p<0.001)$. Caffeine concentrations were higher in the caffeine condition than in the placebo condition for both groups $(\mathrm{F}(1,30)>60.0 ; p<0.001$ for both groups). In the placebo condition, caffeine concentration was higher in the Night group than in the DayRec group $(\mathrm{F}(1,30)=17.9$; $p<0.001)$, while caffeine concentration did not differ between the two groups in the caffeine condition. At wake time, caffeine concentration was higher in the caffeine condition compared to the placebo condition $(\mathrm{F}(1,30)=231.5 ; p<0.001)$, as well as in the Night group compared to the DayRec group $(\mathrm{F}(1,30)=6.8 ; p<0.02)$.

\section{PSG Sleep Variables}

Table 2 presents sleep variables (means and SEM) for the Night and the DayRec groups. F and $p$-values from the Group-by-Condition ANOVAs are also presented. Compared to the Night group, the DayRec group showed reduced sleep latency, less stage 2 sleep ( $\mathrm{min}$ ), and more SWS (\%) in both conditions. Compared to placebo, caffeine administration lengthened sleep latency, increased stage 1 (\%), and reduced stage 2 and SWS ( $\mathrm{min}$ ) both in the Night and DayRec groups. Significant interactions between conditions (caffeine, placebo) and groups (Night, DayRec) were found for total sleep time, number of minutes of wakefulness, sleep efficiency, and REM sleep (min and \%). Contrast analyses showed that caffeine reduced sleep efficiency more strongly in the DayRec group $(\mathrm{F}(1,32)=59.5 ; p<0.0001)$ than in the Night group $(\mathrm{F}(1,32)=4.6 ; p=0.04)$. Compared to placebo, caffeine increased the number of minutes of wakefulness $(\mathrm{F}(1,32)=29.7 ; \quad p<0.001)$, decreased total sleep time $(\mathrm{F}(1,32)=41.8 ; p>0.001)$, and reduced the percentage $(\mathrm{F}(1,32)=3.7 ; p=0.06)$ as well as the number of minutes of REM sleep $(\mathrm{F}(1,32)=12.9 ; p=0.001)$ only in the DayRec group (see Figure 3 ).

Figure 4 illustrates the number of minutes of wakefulness, REM sleep, and SWS per third of the sleep episode, for the two groups as well as the two conditions. A significant interaction between group, condition, and part of the sleep episode was found for the number of minutes of wakefulness $(\mathrm{F}(2,64)=4.5 ; p=0.02 ; \varepsilon=1)$. Simple effects showed that caffeine increased the amount of wakefulness in the first third of the sleep episode $(\mathrm{F}(1,32)=20.9 ; p<0.001)$. In the $2 / 3$ of the sleep episode, there was a significant interaction between group and condition $(\mathrm{F}(1,32)=19.2$;

Table 2 Sleep Variables for the Night and the DayRec Groups in the Placebo and the Caffeine Conditions

\begin{tabular}{|c|c|c|c|c|c|c|c|}
\hline & \multicolumn{2}{|c|}{ Night } & \multicolumn{2}{|c|}{ DayRec } & \multirow[b]{2}{*}{ Group } & \multirow[b]{2}{*}{ Condition } & \multirow[b]{2}{*}{ Interaction } \\
\hline & PLAC & CAF & PLAC & CAF & & & \\
\hline Sleep latency $(\mathrm{min})^{\mathrm{a}}$ & $7.8(1.4)$ & $19.7(4.8)$ & $3.6(1.0)$ & $6.4(1.8)$ & $\begin{array}{c}F(I, 32)=14.6 \\
p<0.001\end{array}$ & $\begin{array}{c}F(1,32)=26.7 \\
p<0.001\end{array}$ & NS \\
\hline REM sleep latency $(\min )^{a}$ & $63.5(3.6)$ & $58.1(4.0)$ & $58.1(9.7)$ & $53(12.4)$ & NS & NS & NS \\
\hline Total sleep time (min) & $426.1(16.0)$ & $401.9(18.6)$ & $346.2(16.3)$ & $264.5(22.7)$ & $\begin{array}{c}F(1,32)=19.3 \\
p<0.001\end{array}$ & $\begin{array}{c}F(1,32)=35.1 \\
p<0.001\end{array}$ & $\begin{array}{c}F(1,32)=10.3 \\
p=0.003\end{array}$ \\
\hline Total awake time $(\mathrm{min})^{\mathrm{a}}$ & $33.0(7.4)$ & $40.4(9.2)$ & $61.7(9.3)$ & | $46.3(22.5)$ & $\begin{array}{c}F(I, 32)=19.6 \\
p<0.001\end{array}$ & $\begin{array}{c}F(I, 32)=27.0 \\
p<0.001\end{array}$ & $\begin{array}{c}F(I, 32)=6.3 \\
p=0.017\end{array}$ \\
\hline Sleep efficiency (\%) & $90.1(1.6)$ & $85.1(2.9)$ & $76.5(3.9)$ & $58.5(5.5)$ & $\begin{array}{c}F(I, 32)=16.2 \\
p<0.001\end{array}$ & $\begin{array}{c}F(I, 32)=48.5 \\
p<0.001\end{array}$ & $\begin{array}{c}F(I, 32)=15.5 \\
p<0.001\end{array}$ \\
\hline Total number of awaking & $24.9(3.2)$ & $31.4(3.5)$ & $36.7(4.9)$ & $35.8(4.6)$ & NS & NS & NS \\
\hline Stage I $(\min )^{a}$ & $28.4(4.5)$ & $32.5(3.7)$ & $25.0(4.0)$ & $26.2(3.9)$ & NS & NS & NS \\
\hline Stage 2 (min) & $279.9(10.2)$ & $253.0(\mid 1.9)$ & $211.9(14.5)$ & | $62.9(\mid 4.7)$ & $\begin{array}{c}F(I, 32)=22.6 \\
p<0.001\end{array}$ & $\begin{array}{c}F(1,32)=24.2 \\
p<0.001\end{array}$ & NS \\
\hline SWS $(\min )^{a}$ & $16.3(5.1)$ & $12.6(3.7)$ & $43.8(10.0)$ & $30.4(8.0)$ & NS & $\begin{array}{c}F(I, 32)=5.4 \\
p=0.03\end{array}$ & NS \\
\hline REM sleep $(\min )^{a}$ & $101.6(7.4)$ & $103.8(7.9)$ & $65.6(5.6)$ & $45.3(6.5)$ & $\begin{array}{c}F(I, 32)=18.2 \\
p<0.001\end{array}$ & $\begin{array}{c}F(I, 32)=6.6 \\
p=0.02\end{array}$ & $\begin{array}{c}F(1,32)=6.3 \\
p=0.02\end{array}$ \\
\hline$\%$ Stage ${ }^{a}$ & $6.6(1.0)$ & $8.2(1.0)$ & $7.4(1.2)$ & $10.3(1.4)$ & NS & $\begin{array}{c}F(I, 32)=16.3 \\
p<0.001\end{array}$ & NS \\
\hline$\%$ Stage $2^{a}$ & $66.0(1.3)$ & $63.3(1.4)$ & $60.9(3.0)$ & $63.6(3.3)$ & NS & NS & NS \\
\hline$\% \mathrm{SWS}^{\mathrm{a}}$ & $3.8(1.2)$ & $3.1(1.0)$ & $12.6(2.8)$ & $10.0(2.5)$ & $\begin{array}{c}F(I, 32)=7.5 \\
p=0.010\end{array}$ & NS & NS \\
\hline$\%$ REM & $23.6(1.3)$ & $25.4(1.4)$ & 19.1 (1.3) & |6.| (I.9) & $\begin{array}{c}F(I, 32)=14.2 \\
p<0.001\end{array}$ & NS & $\begin{array}{c}F(I, 32)=4.6 \\
p=0.04\end{array}$ \\
\hline
\end{tabular}

F and p-values for Group-by-condition ANOVAs are also presented.

asleep parameters that were log transformed before statistical analyses. 

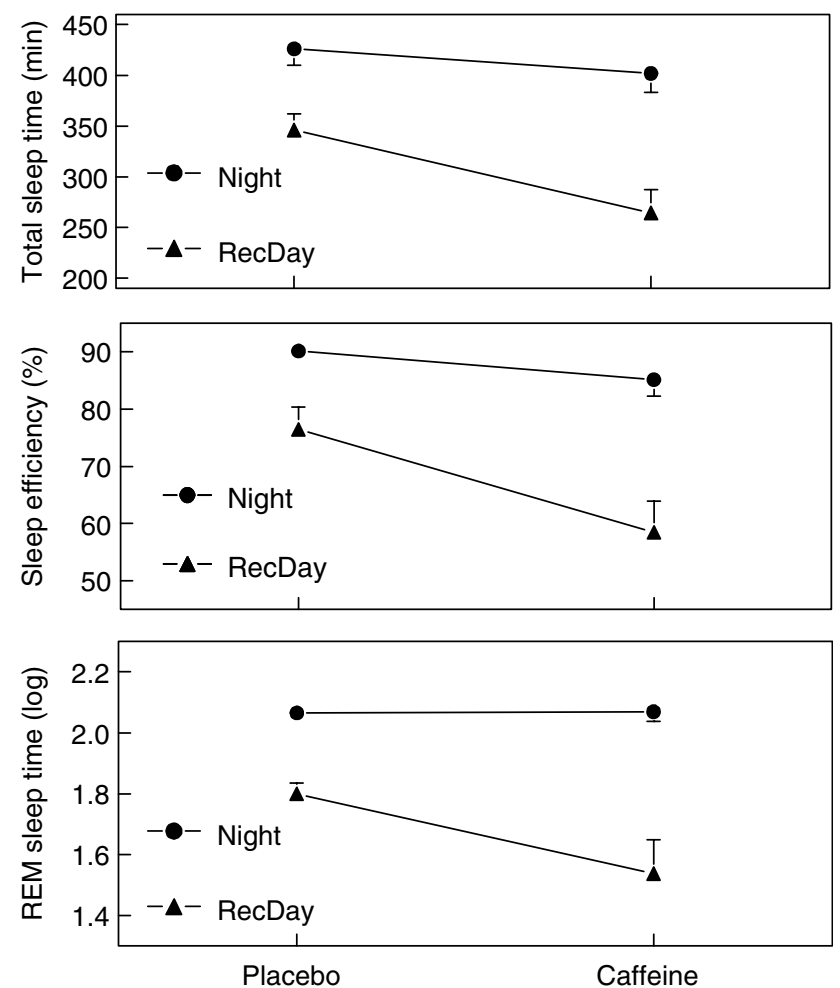

Figure 3 Sleep efficiency, total sleep time and minutes (log) of REM sleep (mean and SEM) for the Night group and the DayRec group, in the placebo and the caffeine conditions.

$p<0.001)$, showing an increase of wakefulness in the caffeine condition in the DayRec group only $(\mathrm{F}(1,32)=$ 46.6; $p<0.001)$. The last third of the sleep episode showed a significant effect for group $(\mathrm{F}(1,32)=19.2 ; p=0.001)$ and condition $(\mathrm{F}(1,32)=10.6 ; p=0.003)$. Number of minutes of wakefulness in the last third of the sleep episode was higher in the caffeine condition compared to the placebo condition, and was higher in the DayRec group compared to the Night group. A significant interaction between group and condition $(\mathrm{F}(1,32)=11.3 ; p<0.002)$, and between group and third of the sleep episode $(\mathrm{F}(2,64)=24.2 ; p<0.001$; $\varepsilon=1$ ) was found for the number of minutes of REM sleep. Only the DayRec group showed a significant decrease of REM sleep following caffeine administration $(\mathrm{F}(1,32)=21.2$; $p<0.001)$. Also, the DayRec group showed less REM sleep than the Night group in the second third $(\mathrm{F}(1,32)=43.5$; $p<0.001)$ and last third $(\mathrm{F}(1,32)=30.4 ; p<0.001)$ of the sleep episode, but not in the first third. A significant interaction between group, condition, and part of the sleep episode was found for the number of minutes of SWS $(\mathrm{F}(2,64)=4.4 ; p=0.02 ; \varepsilon=0.82)$. Simple effects showed that caffeine decreased the amount of SWS in the first third of the sleep episode $(\mathrm{F}(1,32)=6.5 ; p<0.02)$. In the second third of the sleep episode, there was a significant interaction between group and condition $(\mathrm{F}(1,32)=9.9 ; p<0.004)$, showing a decreased amount of SWS only in the DayRec group $(\mathrm{F}(1,32)=11.8 ; p<0.002)$. The last third of the sleep episode showed a significant effect for group $(\mathrm{F}(1,32)=4.7$; $p<0.04)$. Number of minutes of SWS was higher in the last third of the night in the DayRec group compared to the Night group.
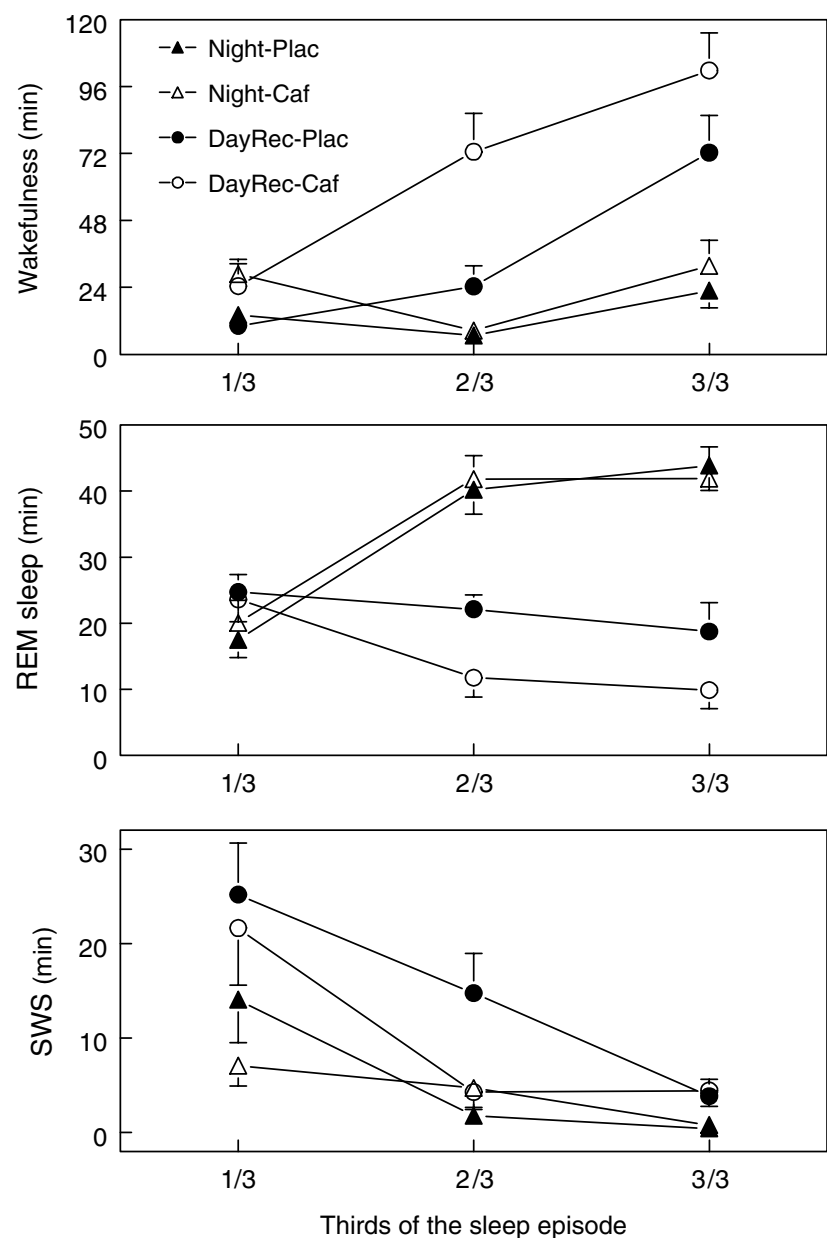

Figure 4 Number of minutes of wakefulness (top panel), REM sleep (middle panel) and SWS (lower panel) per third of the sleep period from sleep onset to lights on (mean and SEM), for the Night group and the DayRec group in the placebo and the caffeine conditions.

\section{DISCUSSION}

Caffeine lengthened sleep latency, increased the percentage of stage 1 sleep, and decreased the number of minutes of stage 2 sleep and of SWS similarly during daytime recovery sleep and nocturnal sleep. The effects of caffeine on such sleep variables have been reported in several studies (Drapeau et al, 2006; Karacan et al, 1976; Landolt et al, 1995). Our study further demonstrates that these effects were not modulated by differences in homeostatic sleep pressure and circadian phase between the two groups. Compared to habitual nocturnal sleep, sleep latency and minutes of stage 2 sleep were reduced and the percentage of SWS was enhanced during daytime recovery sleep, regardless of the condition (placebo or caffeine). These effects are in agreement with previous studies on daytime recovery sleep (Gaudreau et al, 2001; Weitzman and Kripke, 1981), and are explained by differences in homeostatic pressure and circadian phase between the two groups (Achermann et al, 1993; Dijk and Czeisler, 1994).

Importantly, caffeine decreased sleep efficiency more strongly when administered before daytime recovery sleep than before nocturnal sleep. In addition, caffeine reduced 
total sleep time, the number of minutes of wakefulness, and both minutes and percentage of REM sleep only when administered before daytime recovery sleep. The more pronounced effects of caffeine on sleep at a time when the biological clock is sending an increasing waking signal were observed despite the fact that subjects were sleep deprived prior to daytime recovery sleep.

A nonlinear interaction of the circadian and the sleepdependent components of sleep propensity has been reported in forced desynchrony studies (Dijk and Czeisler, 1994). These studies have shown that the last portion of a sleep episode (when homeostatic sleep pressure is low) is more vulnerable to a circadian phase of high wake propensity than is the beginning of a sleep episode (when homeostatic sleep pressure is high). In the present study, daytime recovery sleep episode occurred at a circadian time of increasing wake propensity. The higher amount of wakefulness observed during daytime recovery sleep compared to nocturnal sleep was more prominent at the end of the sleep episode. This is because as the daytime recovery sleep episode progressed, homeostatic sleep propensity decreased and circadian wake propensity increased, leading to more awakenings. Interestingly, the stronger effect of caffeine on the amount of wakefulness during daytime recovery sleep was observed after the first part of the sleep episode. This stronger increase of wakefulness in the DayRec group was accompanied by a reduction of total sleep time at the expense of REM sleep, while caffeine did not influence total sleep time or the amount of REM sleep in the Night group. The decrease of REM sleep in the DayRec group occurred after the first third of the night, when the increase of wakefulness by caffeine was more prominent. The stronger effects of caffeine on daytime recovery sleep compared to nocturnal sleep are probably modulated by the combined influence of increasing circadian wake propensity drive and the dissipation of homeostatic sleep pressure. Further studies using procedures where the number of hours of wakefulness before the sleep episode is kept constant over different circadian phases (eg the forced desynchrony protocol) will be necessary to evaluate the relative contribution of these two factors. One study using forced desynchrony have reported that sleep efficiency might be more affected by caffeine when melatonin secretion is low but complete analysis on sleep were not performed (Wyatt et al, 2004).

The elevated homeostatic sleep pressure induced by sleep deprivation accounts for a higher amount of SWS in daytime recovery sleep compared to night time sleep (Achermann et al, 1993; Gaudreau et al, 2001). Caffeine reduced total amount of SWS in both groups, but more strongly in the second third of the sleep episode in the DayRec group compared to the Night group. The stimulant effects of caffeine on the central nervous system at doses obtained through normal human consumption are due to caffeine's ability to antagonize adenosine receptors (Fredholm et al, 1999). Numerous reports have corroborated that adenosine is a neuromodulator that is involved in sleep regulation (Basheer et al, 2000; Benington et al, 1995; Porkka-Heiskanen et al, 1997; Radulovacki et al, 1982, 1985; Satoh et al, 1996, 1999; Scammell et al, 2001; Schwierin et al, 1996; Strecker et al, 2000; Ticho and Radulovacki, 1991;
Urade et al, 2003). It has recently been proposed that in humans, caffeine mimics the effects of a reduction in sleep homeostasis during prolonged wakefulness (Landolt et al, 2004). Recently, we suggested that the amount of SWS or homeostatic pressure during recovery daytime sleep is critical to 'override' the circadian waking signal (Gaudreau et al, 2001). According to this hypothesis, the daytime circadian signal is more likely to disturb sleep as the homeostatic pressure during daytime recovery sleep is decreased. This interpretation is in line with a recent animal study showing an increase in the firing rate of the suprachiasmatic nucleus during EEG SWS deprivation (Deboer et al, 2003). Accordingly, in our study, the reduction of SWS by caffeine administration was expected to increase the impact of the waking circadian signal on daytime sleep. Our results were consistent with that hypothesis and showed a steep increase in wakefulness in the caffeine condition compared to the placebo condition in the DayRec group, especially in the second third of the sleep episode when the difference in SWS between the two conditions was more prominent. As expected for the Night group, the reduction in SWS by caffeine had less impact on sleep consolidation parameters since sleep occurred at a time when the circadian waking signal was decreasing.

In our study, subjects were instructed to stop using caffeine at noon. Since baseline salivary samples were collected at a later time in the DayRec group (during the night) than in the Night group (during the evening), more time elapsed since the last caffeine consumption in the DayRec group. This explains the lower caffeine levels in the DayRec group than in the Night group (Figure 2). Because of this, the difference at bedtime in caffeine levels between the caffeine and placebo conditions was more prominent in the DayRec group than in the Night group. However, we do not believe that this difference could explain the stronger effects of caffeine on sleep in the DayRec group. As shown in Figure 2, this difference decreased in the course of the sleep episode and the two groups showed at wake time the same difference in caffeine levels between the placebo and the caffeine conditions. This contrasts with the effects of caffeine on sleep parameters, which were similar in the two groups at the beginning of sleep but stronger in the DayRec group in the later parts of the sleep episode.

In conclusion, the effects of caffeine on sleep consolidation and REM sleep are more prominent when caffeine is consumed before daytime recovery sleep than before nocturnal sleep. The stronger effects of caffeine on daytime recovery sleep compared to nocturnal sleep are probably the consequence of the combined influence of increasing circadian wake propensity drive and the dissipation of homeostatic sleep pressure in the course of the sleep episode. We propose that the reduction of SWS by caffeine during daytime sleep increases the impact of the circadian wake signal on sleep. Recent studies have suggested that caffeine is a measure of choice to counteract the effects of sleep deprivation on alertness and performance (Beaumont et al, 2005). Our results suggest that this recommendation should be made with cautious since using caffeine before sleeping at an abnormal circadian phase, such as during night work, might have more adverse consequences on sleep quality than before sleeping at a normal circadian time. 


\section{ACKNOWLEDGEMENTS}

This research was supported by scholarships and grants from the Canadian Institutes of Health Research (CIHR), the Fonds de Recherche en Santé du Québec (FRSQ), and the Natural Sciences and Engineering Research Council of Canada (NSERC). We thank Sonia Frenette (project coordinator), our technicians for day-to-day study management, and Valérie Mongrain, Catherine Beaulieu, and Hélène Blais for thoughtful comments on the manuscript.

\section{REFERENCES}

Achermann P, Dijk D-J, Brunner DP, Borbély A (1993). A model of human sleep homeostasis based on EEG slow-wave activity: quantitative comparison of data and simulations. Brain Res Bull 31: 97-113.

Akerstedt T (1984). Work schedules and sleep. Experientia 40: 417-422.

Alkaysi HN, Shiekh MS, el-Sayed YM (1988). High performance liquid chromatographic analysis of caffeine concentrations in plasma and saliva. J Clin Pharm Ther 13: 109-115.

Barone JJ, Roberts HR (1996). Caffeine consumption. Food Chem Toxicol 34: 119-129.

Basheer R, Porkka-Heiskanen T, Strecker RE, Thakkar MM, McCarley RW (2000). Adenosine as a biological signal mediating sleepiness following prolonged wakefulness. Biol Signals Receptor 9: 319-327.

Beaumont M, Batéjat D, Coste O, Doireau P, Chauffard F, Enslen $M$ et al (2005). Recovery after prolonged sleep deprivation: residual effects of slow-release caffeine on recovery sleep, sleepiness and cognitive functions. Neuropsychobiology 51: 16-27.

Benington JH, Kodali SK, Heller HC (1995). Stimulation of A1 adenosine receptors mimics the electroencephalographic effects of sleep deprivation. Brain Res 692: 79-85.

Bonnet MH, Arand DL (1996). Metabolic rate and the restorative function of sleep. Physiol Behav 59: 777-782.

Borbély AA, Achermann P (2000). Sleep homeostasis and models of sleep regulation. In: Kryger MH, Roth T, Dement WC (eds). Principles and Practice of Sleep Medicine. WB Sauders Company: Philadelphia. pp 377-390.

Brezinova V (1974). Effects of caffeine on sleep: EEG study in late middle age people. Br J Clin Pharmacol 1: 203-208.

Broughton RJ (1998). SCN controlled circadian arousal and the afternoon 'nap zone'. Sleep Res Online 1: 166-178.

Daan S, Beersma DGM, Borbely AA (1984). Timing of human sleep: recovery process gated by circadian pacemaker. Am J Physiol 246: R161-R183.

Deboer T, Vansteensel MJ, Détari L, Meijer JH (2003). Sleep states alter activity of suprachiasmatic nucleus neurons. Nat Neurosci 6: $1086-1090$.

Dijk DJ, Czeisler CA (1994). Paradoxical timing of the circadian rhythm of sleep propensity serves to consolidate sleep and wakefulness in humans. Neurosci Lett 166: 63-68.

Drapeau C, Hamel-Hebert I, Robillard R, Selmaoui B, Filipini D, Carrier J (2006). Challenging sleep in aging: the effects of $200 \mathrm{mg}$ of caffeine during the evening in young and middle-aged moderate caffeine consumers. J Sleep Res 15: 133-141.

Fredholm BB, Bättig K, Holmén J, Nehlig A, Zvartau EE (1999). Actions of caffeine in the brain with special reference to factors that contribute to its widespread use. Pharmacol Rev 51: 83-133.

Gaudreau H, Morettini J, Lavoie HB, Carrier J (2001). Effects of a 25-h sleep deprivation on daytime sleep in the middle-aged. Neurobiol Aging 22: 461-468.
Karacan I, Thornby JI, Anch AM, Booth GH, Williams RL, Salis PJ (1976). Dose-related sleep disturbances induced by coffee and caffeine. Clin Pharmacol Ther 20: 682-689.

Kelly TL, Mitler MM, Bonnet MH (1997). Sleep latency measures of caffeine effects during sleep deprivation. Electroencephalogr Clin Neurophysiol 102: 397-400.

LaJambe CM, Kamimori GH, Belenky G, Balkin TJ (2005). Caffeine effects on recovery sleep following $27 \mathrm{~h}$ total sleep deprivation. Aviat Space Environ Med 76: 108-113.

Landolt HP, Dijk D-J, Gaus SE, Borbély AA (1995). Caffeine reduces low-frequency delta activity in the human sleep EEG. Neuropsychopharmacology 12: 229-238.

Landolt HP, Rétey JV, Tönz K, Gottselig JM, Khatami R, Buckelmüller I et al (2004). Caffeine attenuates waking and sleep electroencephalographic markers of sleep homeostasis in humans. Neuropsychopharmacology 29: 1933-1939.

Monk TH, Reynolds CFI, Kupfer DJ, Buysse DJ, Coble PA, Hayes AJ et al (1994). The Pittsburgh sleep diary. J Sleep Res 3: 111-120.

Muehlbach MJ, Walsh JK (1995). The effects of caffeine on simulated night-shift work and subsequent daytime sleep. Sleep 18: 22-29.

Porkka-Heiskanen T, Strecker RE, Bjorkum AA, Thakkar M, Greene RW, McCarley RW (1997). Adenosine: a mediator of the sleep-inducing effects of prolonged wakefulness. Science 276: 1265-1268.

Radulovacki M (1985). Role of adenosine in sleep in rats. Rev Clin Basic Pharmacol 5: 327-339.

Radulovacki M, Miletich RS, Green RD (1982). $N^{6}$ (L-phenylisopropyl) adenosine (L-PIA) increases slow-wave sleep $\left(\mathrm{S}_{2}\right)$ and decreases wakefulness in rats. Brain Res 246: 178-180.

Rechtschaffen A, Kales AA (1968). A Manual of Standardized Terminology, Techniques, and Scoring System for Sleep Stages of Human Subjects. National Institute of Neurological Diseases and Blindness: Bethesda, MD.

Reyner LA, Horne JA (2000). Early morning driver sleepiness: effectiveness of $200 \mathrm{mg}$ caffeine. Psychophysiology 37: 251-256.

Satoh S, Matsumura H, Koike N, Tokunaga Y, Maeda T, Hayaishi O (1999). Region-dependent difference in the sleep-promoting potency of an adenosine A2a receptor agonist. Eur J Neurosci 11: 1587-1597.

Satoh S, Matsumura H, Suzuki F, Hayaishi O (1996). Promotion of sleep mediated by the A2a-adenosine receptor and possible involvement of this receptor in the sleep induced by prostaglandin D2 in rats. Proc Natl Acad Sci USA 93: 5980-5984.

Scammell TE, Gerashchenko DY, Mochizuki T, McCarthy MT, Estabrooke IV, Sears CA et al (2001). An adenosine A2a agonist increases sleep and induces fos in ventrolateral preoptic neurons. Neuroscience 107: 653-663.

Schwierin B, Borbély AA, Tobler I (1996). Effects of $N^{6}$ cyclopentyladenosine and caffeine on sleep regulation in the rat. Eur J Pharmacol 300: 163-171.

Strecker RE, Morairty S, Basheer R, Thakkar MM, PorkkaHeiskanen T, Dauphin LL et al (2000). Adenosinergic modulation of basal forebrain and preoptic/anterior hypothalamic neuronal activity in the control of behavioral state. Behav Brain Res 115: 183-204.

Ticho SR, Radulovacki M (1991). Role of adenosine in sleep and temperature regulation in the preoptic area of rats. Pharmacol Biochem Behav 40: 33-40.

Urade Y, Eguchi N, Qu WM, Sakata M, Huang ZL, Chen JF et al (2003). Sleep regulation in adenosine A2a receptor-deficient mice. Nippon Yakurigaku Zasshi 61: S94-S96.

Van Dongen HPA, Price NJ, Mullington JM, Szuba MP, Kapoor SC, Dinges DF (2001). Caffeine eliminates psychomotor vigilance deficits from sleep inertia. Sleep 24: 813-819. 
Walsh JK, Muehlbach MJ, Humm TM, Dickins QS, Sugerman JL, Schweitzer PK (1990). Effect of caffeine on physiological sleep tendency and ability to sustain wakefulness at night. Psychopharmacology 101: 271-273.

Warburton DM (1995). Effects of caffeine on cognition and mood without caffeine abstinence. Psychopharmacology 119: 66-70.

Weitzman ED, Kripke DF (1981). Experimental 12-h shift of the sleep-wake cycle in man: Effects on sleep and physiological rhythms. In: Johnson LC, Tepas DI, Colquhoun WP, Colligan MJ (eds). Biological Rhythms, Sleep and Shift Work. Spectrum Publishing: New York. pp 93-110.

Wurts SW, Edgar DM (2000). Caffeine during sleep deprivation: sleep tendency and dynamics of recovery sleep in rats. Pharmacol Biochem Behav 65: 155-162.

Wyatt JK, Cajochen C, Ritz-De Cecco A, Czeisler CA, Dijk DJ (2004). Low-dose repeated caffeine administration for circadianphase-dependent performance degradation during extended wakefulness. Sleep 27: 374-381. 